\title{
Simulative Untersuchungen des Einflusses elektromechanischer Belastungen an einem Axiallager auf die Glättung der Oberflächen und den Traganteil im Kugel-Laufbahnkontakt
}

\author{
Simon Graf' ${ }^{1} \mathbb{D} \cdot$ Bernd Sauer ${ }^{1}$ \\ Eingegangen: 1. März 2021 / Angenommen: 27. Januar 2022 / Online publiziert: 25. Februar 2022 \\ ○ Der/die Autor(en) 2022
}

\section{Zusammenfassung}

Es werden Ergebnisse aus einer Kontaktsimulation vorgestellt, welche die Oberflächenveränderung eines Axiallagers infolge von unerwünschtem elektrischem Stromdurchgang bei Mischreibung zeigen. Das hierzu entwickelte Modell berücksichtigt neben den Oberflächenrauheiten auch das nichtlineare Materialverhalten des Wälzlagerwerkstoffes. Im Gegensatz zu bekannten Modellierungsmethoden für ähnliche Problemstellungen, wird hier ein neuartiger Ansatz auf Basis einer gekoppelten Euler- Lagrange- Finite Element Simulation entwickelt. Das Modell liefert, mit experimentell geschädigten Oberflächen als Eingangsgröße, Erkenntnisse zum Traganteilsverhalten und weiterer mechanischer Kenngrößen infolge kombinierter mechanischer und elektrischer Belastungen.

\section{Simulative investigations of the influence of electromechanical loads on a thrust bearing on the smoothing of the surfaces and the contact ratio in the ball/raceway contact}

\begin{abstract}
Results from a contact simulation are presented, which show the surface change of an axial roller bearing due to undesired electric current passage under mixed friction. The model developed for this purpose takes into account not only the surface roughness but also the nonlinear material behavior of the rolling bearing material. In contrast to known modeling methods for similar problems, a novel approach based on a coupled Eulerian-Lagrangian finite element simulation is developed. Using experimentally damaged surfaces as input, the model provides insights into the load-bearing behavior and other mechanical parameters as a result of combined mechanical and electrical loads.
\end{abstract}

\section{Einleitung}

Durch elektrischen Stromdurchgang an Wälzlagern induzierte Schäden, wie beschädigte Laufbahnen und verfärbte Schmiermittel, wurden bereits ab 1907 unter anderem durch Punga und Hess [1], Fleischmann [2] und Kellner [3] beschrieben. Auf diesen Arbeiten aufbauend wurden konstruktive Maßnahmen abgeleitet, wodurch die Lagerstromproblematik zunächst beseitigt werden konnte. Mit

Simon Graf

s.graf@mv.uni-kl.de

1 Lehrstuhl für Machinenelemente und Getriebetechnik (MEGT), Technische Universität Kaiserslautern, Gottlieb Daimler Straße 42, 67663 Kaiserslautern, Deutschland dem Einzug von hochfrequenten Frequenzumrichtern und dem damit verbundenen vermehrten Aufkommen von drehzahlvariablen Antriebskonzepten, kam es zu einem erneuten Auftreten von unerwünschten Lagerströmen (u.a. [4, 5]). Ursächlich hierfür sind die für diesen Typus von Frequenzumrichter typischen steilen Spannungsflanken in Kombination mit ungünstig konstruierten Erdungskonzepten, wodurch die bereits in [1] beschriebenen Schäden, wieder auftreten können [6-8]. Bei der Art des auftretenden Schädigungsphänomens spielt die Wechselwirkung zwischen dem tribologischen System definiert durch den Schmierungs/und Reibungszustand und dem sich daraus definierten elektrischen Verhalten, eine entscheidende Rolle.

Der kausale Zusammenhang zwischen dem im Wälzlager vorhandenen Schmierungszustand und der Art der sich 


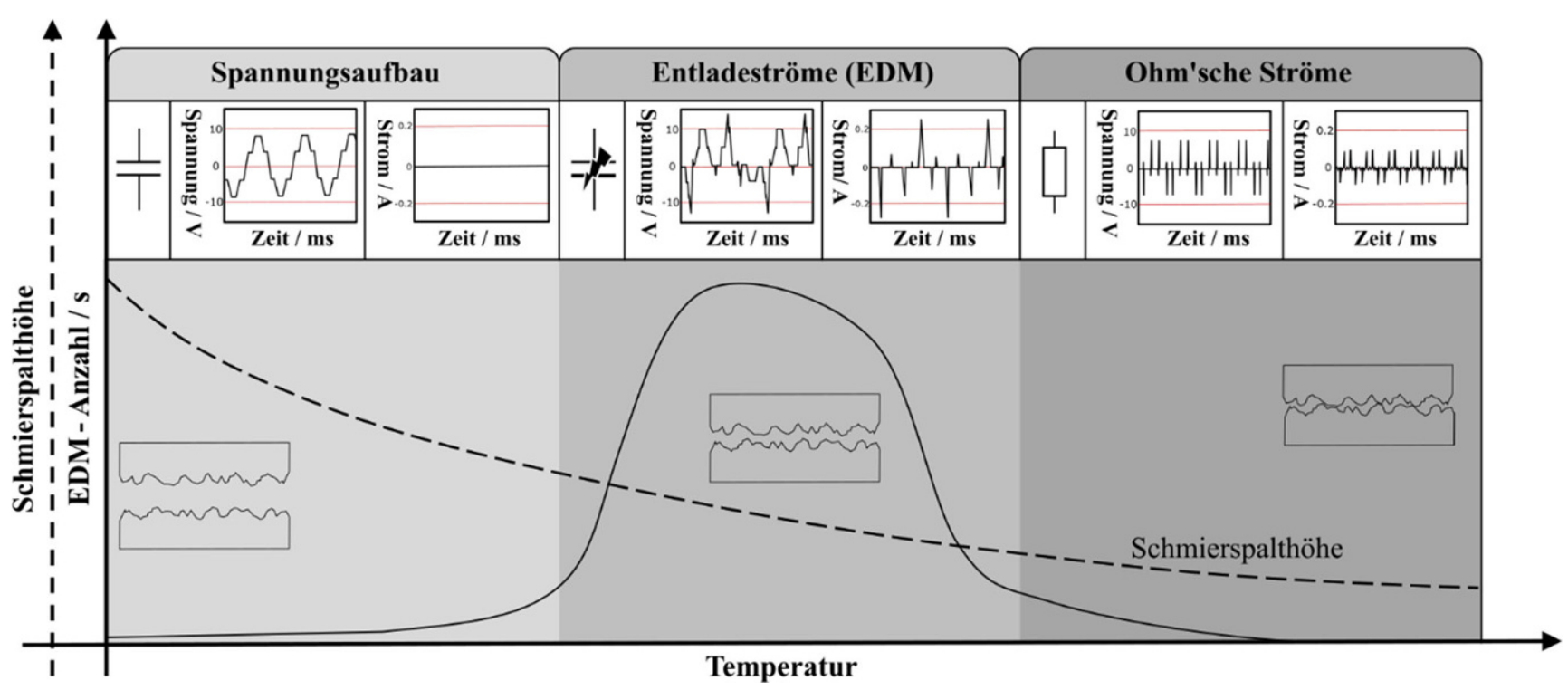

Abb. 1 Zonen abhängige Darstellung der EDM-Anzahl pro Sekunde bei Schmierfilmdickenvariation infolge von Temperatursteigerung bei konstanter Prüfspannung sowie Darstellung des elektrischen Ersatzsystems mit den jeweiligen charakteristischen Spannungs- und Stromverläufen (exemplarisch)

einstellenden Lagerströme wurde in [9] aufgezeigt und lässt sich anhand Abb. 1 anschaulich visualisieren.

Hier wird die Abhängigkeit der Häufigkeit des Auftretens von Entladeströmen, sogenannten EDM Strömen (engl. Electic Discharge Maching), von der Temperatur bei ansonsten konstanten mechanischen Randbedingungen und gleichbleibender Spannung gezeigt. Die Erhöhung der Temperatur im ansonsten konstanten System geht mit einer Reduzierung der Viskosität des Schmierstoffes und damit mit einer Variation der Schmierspalthöhe einher. Somit geht das untersuchte Wälzlager vom Vollschmierungszustand in den Mischreibungszustand über, was mittels des exemplarischen Schmierspaltverlaufs und den visualisierten Rauheiten im Diagramm verdeutlicht wird. Das Wälzlager als elektrisches Bauteil betrachtet ist von dieser Änderung ebenfalls betroffen. So kann bei ausreichender Schmierfilmhöhe der Wälzkontakt zunächst als Kondensator beschrieben werden. Hierin sind die Kontaktflächen des Wälzkörpers und der Wälzkörperlaufbahn die Elektroden und das verwendete Schmiermittel das Dielektrikum. Bei ausreichender Schmierfilmhöhe liegt zunächst ein isolierendes, kapazitives Verhalten des Lagers vor. Das bedeutet, dass die aufgeprägte Common Mode Spannung, charakterisiert durch das Aufladen des Kondensators mittels dreier Spannungsimpulse gefolgt von drei Entladeimpulsen in identischer Höhe, vollständig im Kondensator gehalten werden kann (vgl. Abb. 1 Spannungsaufbau ohne EDM Entladungen). Durch eine einsetzende Schmierfilmdickenreduzierung, beispielsweise infolge einer Temperaturerhöhung, reduziert sich der Abstand der Elektroden (Wälzkörper zu Wälzkörperlaufbahn) des Kondensators zueinander und die anliegende Spannung kann nicht mehr vollständig gehalten werden. Infolge dessen kommt es zu Entladeströmen in Form von Funkenschlägen zwischen den Laufbahnen und dem Wälzkörper. Dieses Verhalten zeigt sich darin, dass ein dreistufiger Aufbau der Common Mode Spannung nicht mehr vollständig möglich ist und es zu einem Zusammenbrechen der Spannung kommt, in deren Folge ein Strom über das Lager fließt (vgl. Abb. 1 Entladeströme). Mittels weiterer Schmierfilmreduzierung tritt das Lager in den tribologischen Zustand der Mischreibung ein. Aus elektrischer Sicht bedeutet dieser Zustand, dass die auftretenden Entladeströme nun nicht mehr den Kondensator aufladen oder überladen, sondern die auftretenden Ströme über die sich nun berührenden Rauheitsspitzen in Form von ohm'schen Strömen abgeleitet werden. Ein Aufbau der elektrischen Spannung ist in diesem Zustand nicht mehr möglich (vgl. Abb. 1 Ohm'sche Ströme).

Experimentelle Ergebnisse aus [10] und [11] zeigten, dass die unerwünschte elektrische Belastung in diesem tribologischen Zustand der Mischreibung zu einem Einglätten der Oberflächen und damit einer Steigerung des Festkörpertraganteils führt. Der Festkörpertraganteil beschreibt das Verhältnis der scheinbaren bzw. nominellen Kontaktflächen zur realen bzw. tragenden Fläche zweier Körper. Der maßgebliche Unterschied hierbei liegt in der Betrachtungsweise der Oberflächen; ob diese als ideal glatte $(\rightarrow$ scheinbare/nominelle Kontaktfläche) oder als real raue $(\rightarrow$ reale/ tragende Kontaktfläche) Kontaktpartner behandelt werden [12].

Die hier vorgestellten Untersuchungen haben zum Ziel die Oberflächenveränderung und damit die Traganteilsstei- 
gerung infolge der elektrischen Belastung bei Mischreibung zu bewerten und vergleichbar zu machen. Hierzu wird zunächst eine Kontaktsimulation einer rein mechanisch belasteten Referenzoberfläche durchgeführt, um die hier auftretende Überrollglättung im Gegensatz zu der Glättung durch die elektrische Belastung zu verdeutlichen. Hierauf aufbauend werden Kontaktsimulationen von solchen Oberflächen durchgeführt, bei welchen elektrische und mechanische Belastungen überlagert wurden. Ausgewertet werden für die jeweiligen Simulationen unter anderem Traganteilskurven. Aus deren Gegenüberstellung kann dann eine exakte Beurteilung der Traganteilssteigerung und der damit einhergehenden Veränderung der tribologischen Bedingungen im Wälzkontakt erfolgen.

Im Kontext des elektrischen Stromdurchgangs ist der Festkörpertraganteil für zwei wesentliche Fragestellungen interessant. Zum einen kann über ihn das vorherrschende tribologische System und damit das sich bildende elektrische System unter Zuhilfename von tribologischen Simulationen identifiziert werden. Zum anderen kann über den Traganteil auch der elektrische Kontaktwiderstand, beispielsweise zwischen Wälzkörper und Wälzlagerlaufbahn bestimmt werden [13]. Dieser Kontaktwiderstand ist wiederum maßgeblich für die elektrische Verlustleistung im Wälzkontakt verantwortlich, womit eine zusätzlich wirkende thermische Beanspruchung des Kontaktes induziert wird.

Klassischerweise wird für die Traganteilssimulation von rauen Oberflächen auf die so genannte Halbraumtheorie zurückgegriffen. Hierbei handelt es sich um ein Verfahren, welches auf den Gleichungen nach Bussinesq [14] basiert und eine Erweiterung einfacher Federmodelle darstellt. Der Vorteil gegenüber alternativen Simulationsmethoden, wie etwa der Finite Elemente Methode (FEM), liegt darin, dass zur Abbildung der Deformations-/Kraft Beziehung eine Dimension weniger modelliert werden kann. In Folge dessen liegen hier auch keinen Vernetzungsunstetigkeiten unter Oberfläche wie etwa bei Lagrangen FE vor. Die Span- nungsverteilung in Tiefenrichtung des Materials wird demnach erst in einem zweiten Berechnungsschritt ermittelt. Dies führt zu einem wesentlichen Vorteil in der Rechenzeit gegenüber einem Finite Elemente Ansatz [15]. Weitere verbreitete Simulationsmethoden zur Abbildung von Kontakten unter Berücksichtigung der Oberflächenstruktur der Kontaktpartner, sind etwa die Randelementemethode oder auch zelluläre Automaten [17]. Bei der Abbildung von nichtlinearem Materialverhalten muss bei Halbraummodellen während der Simulation, die für die Spannungsverteilung notwendige Einflusszahlenmatrix, ermittelt werden und eine Rückrechnung auf die Steifigkeit erfolgen. Abhängig von der sich ergebenden Spannungsausbreitung muss dann die Berechnung der Deformations-/Kraft Beziehung wiederholt durchgeführt werden. Hierdurch wird der bei linear elastischen und elastisch ideal plastischen Materialverhalten numerische Vorteil wie in u. a. [16] dargestellt, gegenüber der Finite Elemente Methode nivelliert.

Weswegen für die hier durchgeführten Kontaktsimulationen ein Finite Elemente Ansatz auf Basis der gekoppelten Euler-Lagrange-Methode (engl. Coupled Eulerian Lagrange (CEL)) auf die vorliegende Problemstellung adaptiert wird. Diese Methode kombiniert die Diskretisierungsarten nach Lagrange und Euler innerhalb eines Modells, wobei Kontinua als Lagrange-Körper formuliert und mit einem in einem Eulernetz diskretisierten Körper interagieren können. Bei der Diskretisierung einer rauen Oberfläche mittels des klassischen Lagrangen Ansatzes kann es zu Vernetzungsunstetigkeiten und stark deformierten Elementen unter Last als Folge der Rauheit kommen (vgl. Abb. 2a). Hierdurch sinkt die Berechnungsgüte bei gleichzeitiger Steigerung der Simulationsdauer. Diese Nachteile können durch die Verwendung eines raumfesten Eulergitters, in welches ein Volumenausschnitt der rauen Oberfläche eingeprägt wird, vermieden werden (vgl. Abb. 2b). Der Vorteil dieser Methode, im Vergleich zu den bekannten Halbraummodellen, liegt im Berechnungsaufwand bei der Abbildung von nichtlinea-
Abb. 2 Darstellung einer exemplarischen Rauheitsspitze zur bespielhaften Verdeutlichung der Diskretisierung mittels a Lagrange-Methode b Euler-Methode

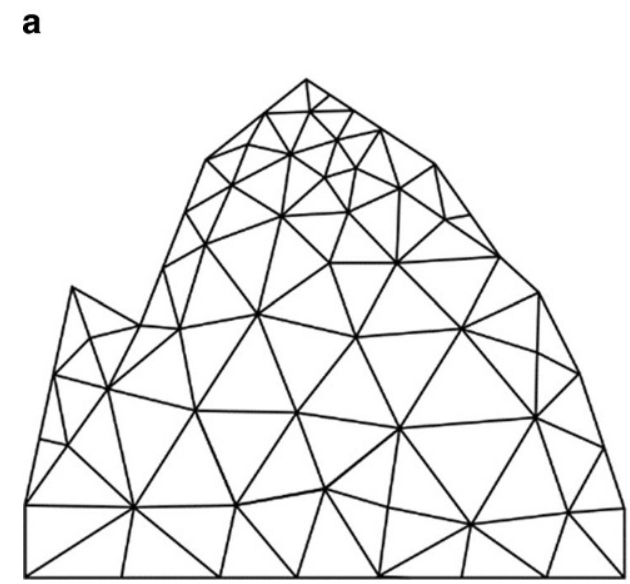

b

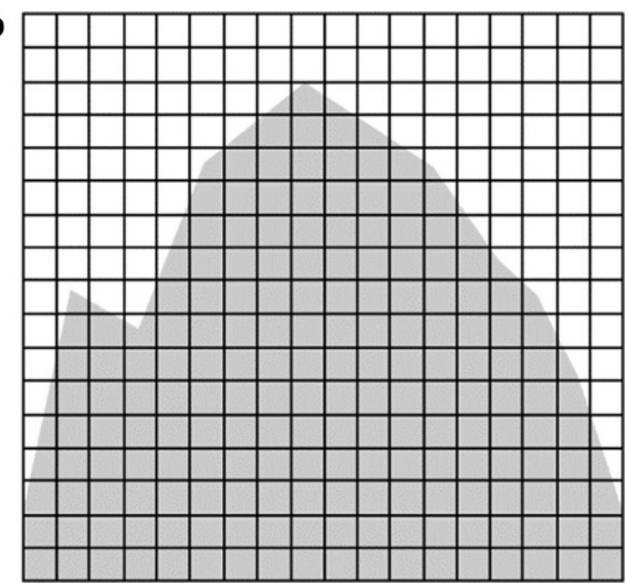


rem Materialverhalten. So muss bei einsetzender Plastifizierung in Halbraummodellen die Einflusszahlenmatrix kontinuierlich angepasst und die Deformations-/Kraft Beziehung iterativ gelöst werden, was mit einem hohen numerischen Aufwand einhergeht [18]. Diese Neugenerierung des zu lösenden Gleichungssystems entfällt bei der Verwendung von FEM. Hier wird lediglich die Elementsteifigkeitsmatrix angepasst. Die Iteration der Deformations-/Kraft Beziehung muss auch hier durchgeführt werden, welche jedoch im Rahmen der Kontaktfindung bereits erfolgt und somit keinen wesentlichen Berechnungsmehraufwand darstellt. Aufgrund dieser Unterschiede zu den gängigen Modellierungsansätzen können mittels der hier vorgestellten Modellierung (Abschn. 3.2) auch relativ große Diskretisierungsbereiche mit adäquater Netzdichte effizient berechnet werden.

Bei dieser Methode (CEL) handelt es sich um eine in anderen Bereichen bewährte Simulationsmethode, welche vor allem zur Simulation von Prozessen verwendet wird, bei denen Bauteile großen Verformungen unterliegen und ein Ergebnis mit akzeptabler numerischer Güte durch eine reine Lagrange-Formulierung nicht mehr erreicht werden kann. Prozesse auf die, die CEL Formulierung angewandt werden kann, können z.B. Tiefziehen [19], Bohren [20], Drehen [21], Rührreibschweißen [22] oder das Thermofügen von Kunststoffen [23] sein.

Auch bei der CEL Methode existieren Anwendungsgrenzen beziehungsweise Besonderheiten, welche beim Aufbau des Modells berücksichtigt werden müssen. So ist etwa die Kontaktfindung numerisch aufwändiger, als bei Simulation basierend auf der Lagrangen Betrachtungsweise. In der verwendeten Softwareumgebung wird diese Problematik mittels einer erweiterten Penalty Methode gelöst [24]. Weiterhin muss berücksichtigt werden, dass es bei der Überführung der Geometrie in ein Eulernetz zu geringen $\mathrm{Ab}$ weichungen in der Oberflächenstruktur kommen kann [25]. Tiefergehende Erläuterungen zur CEL Methode bzw. der eulerschen Beschreibungsform können unteranderem [24, 26] entnommen werden.

\section{Experimentell gewonnene Ergebnisse}

In [10] und [11] wurden Versuchsergebnisse dargestellt, die die Auswirkung des elektrischen Stromdurchgangs auf die Oberflächenveränderung in Axiallagern $(51208 \mathrm{~d} / \mathrm{D} / \mathrm{H}$ 40/68/19) im Laufe der Versuchszeit dokumentieren und untersuchen. Hierbei wurde darauf geachtet, dass über der Versuchszeit überwiegend ohm'sche Ströme, wie sie bei einem Betrieb des Lagers in Mischreibung zu erwarten sind, auftreten. Das Prüflager wird hierzu in einer eigens entwickelten Prüfapparatur, dem sogenannten Gerät zur erweiterten Schmierstoffanalyse (GESA), einer kombinierten mechanisch elektrischen Beanspruchung ausgesetzt. Weiterhin wird zu definierten Versuchszeitpunkten das Prüflager ausgebaut und die Oberflächen der Lagerlaufbahnen mittels eines Konfokalmikroskop vermessen um die Entwicklung der Oberflächenveränderung über der Versuchszeit zu beobachten.

Bei dem GESA (entwickelt bei Forschungsarbeiten zum Projekt FVA 650 II [27]) handelt es sich um einen Prüfstandsadapter, welcher in den Prüfraum eines handelsüblichen Vierkugelapparats eingebaut werden kann. Hierbei werden die mechanischen Randbedingungen, wie Kraft und Drehzahl, über die Belastungsvorrichtungen des Vierkugelapparats aufgebracht. Die elektrischen Randbedingungen werden mittels eines vom Antrieb des Vierkugelapparates unabhängigen Umrichter auf den Prüfling appliziert. Somit ist es möglich, die mechanischen und elektrischen Randbedingungen unabhängig voneinander zu variieren. In u.a. [27, 28] und [29] wurde dieser Prüfaufbau dazu eingesetzt, Schmierstoffe elektrisch zu vermessen und zu charakterisieren.

\subsection{Experimentelle Randbedingungen}

Mechanische Last Mit dem modifizierten Vierkugelapparat wird eine axiale mechanische Belastung von $2400 \mathrm{~N}$ und eine Drehzahl von 1000 U/min auf das Axiallager ausgeübt. Daraus ergibt sich ein maximaler Hertz'scher Kontatkdruck von $\sim 1260 \mathrm{MPa}$ bei einer Kontaktfläche je Kontakt von $\mathbf{0 , 1 9} \mathbf{~ m m}^{2}$ (bei einer Wälzkörperanzahl von $14 \mathrm{Stk} /$ einem Laufbahnprofildurchmesser von $11,1 \mathrm{~mm} /$ und einem Wälzkörperdurchmesser von 10,3 mm). In Kombination mit dem verwendeten Schmierstoff führen diese Bedingungen zu einem Betrieb des Wälzlagers in Mischreibung.

Schmiermittel Zur Schmierung wurde das Mineralöl OF1.1 (Dichte $0,887 \mathrm{~g} / \mathrm{cm}^{3}$, kinetische Viskosität $372,3 \mathrm{~mm}^{2} / \mathrm{s}$, beides bei $20^{\circ} \mathrm{C}$ ) aus dem Forschungsprojekt FVA 650 II [27] verwendet. Hierbei handelt es sich um ein nicht additiviertes Mineralöl, weswegen starke Wechselwirkungen zwischen Versuchsöl und Oberfläche, wie sie etwa bei einem additivierten Hochleistungsschmiermittel zu erwarten sind und zu einer Ausbildung von tribologisch aktiven Schichten führen, nicht zu erwarten sind.

Elektrische Belastung Mittels eines Umrichters werden variable Lagerströme generiert, welche unabhängig vom Umrichter der Antriebseinheit direkt auf den Prüfling aufgeprägt werden können. Durch diese Methodik ist es möglich, die mechanische und elektrische Belastung des Prüflings unabhängig voneinander zu variieren. Weiterhin kann im Gegensatz zum realen Asynchronmotor die auf das Prüflager aufgeprägte Lagerzwischenkreisspannung (bis $60 \mathrm{~V}$ ), die Schaltfrequenz (bis $50 \mathrm{kHz}$ ) und die elektrische Grundfrequenz der pulsweiten modulierten Spannung (bis $100 \mathrm{~Hz}$ ) 
Tab. 1 Elektrische Versuchsmatrix mit elektrischen Beanspruchungsparametern und Benennung der Komponenten gemäß [11]

\begin{tabular}{llll}
\hline Benennung & $20 \mathrm{~V} 72 \mathrm{~h}$ & $40 \mathrm{~V} 72 \mathrm{~h}$ & $60 \mathrm{~V} 72 \mathrm{~h}$ \\
\hline Lagerzwischenkreisspannung/V & 20 & 40 & 60 \\
Lagerspannung/V & 3,3 & 6,6 & 10,0 \\
Lagerstrom/A & 0,2 & 0,4 & 0,6 \\
Lagerstromdichte/A/mm ${ }^{2}$ & 0,075 & 0,15 & 0,225 \\
Lagerscheinleistung/VA & 0,66 & 2,64 & 6 \\
\hline
\end{tabular}

voneinander unabhängig variiert werden. Somit ist es möglich den Prüfling mit sich unterschiedlich ausprägenden Lagerströmen, unabhängig vom betrachteten mechanischen System, zu beaufschlagen. Für die Versuche wurde eine Schaltfrequenz von $10 \mathbf{~ k H z}$ bei einer elektrischen Grundfrequenz von $50 \mathrm{~Hz}$ gewählt. Die Lagerzwischenkreisspannung wurde über einen Versuchslauf je Prüfling konstant gehalten. Je Prüflauf wurden die jeweiligen Prüflager dann mit den elektrischen Belastungen von $0 \mathrm{~V} / 20 \mathrm{~V} / \mathbf{4 0} \mathrm{V}$ und $60 \mathrm{~V}$ beaufschlagt. Die sich einstellenden Lagerspannungen und Lagerströme sind in Tab. 1 zusammengefasst. Weiterhin werden hier übliche Bewertungskennwerte für die elektrische Lagerbelastung, wie die scheinbare Lagerstromdichte konform zu [7] und die Lagerscheinleistung nach [30] für die jeweiligen Lastniveaus angegeben.

\subsection{Ergebnisse}

Für einen ersten Überblick wurden aus den vermessenen Oberflächen charakteristische Oberflächenparameter ( $\mathrm{Sa}$ mittlere arithmetische Höhe/ $S q$ mittlere quadratische Höhe) mittels der Auswertesoftware MountainsMap ${ }^{\circledR}$ bestimmt. Diese sind über der Versuchszeit in Abb. 3 dargestellt. Die hervorgehobenen Punkte und Rechtecke stellen hierbei reale Messpunkte dar. An diesen wurde der Versuch

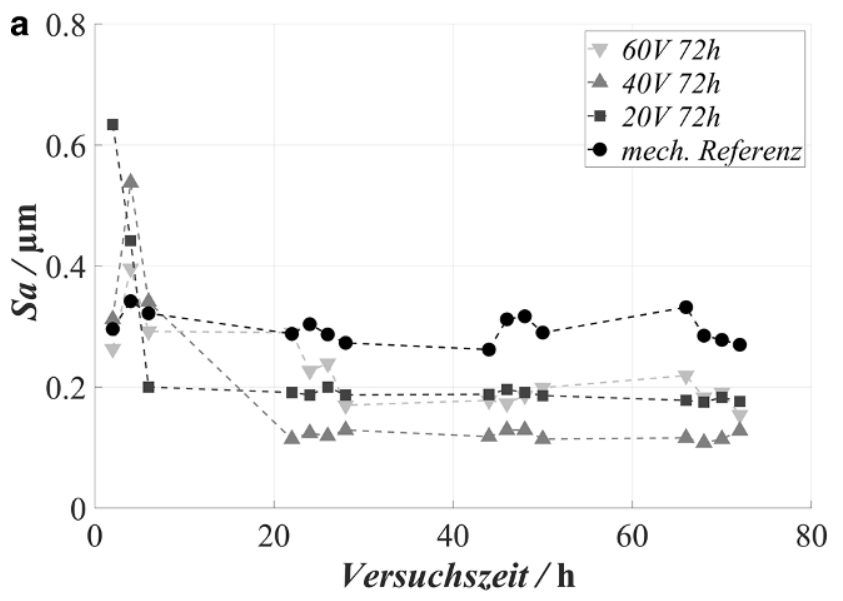

unterbrochen, der stehende Ring gereinigt/vermessen/mit frischem Schmierstoff benetzt/montiert und der Prüflauf weitergeführt. In Abb. 3 zeigt sich, dass die mittlere arithmetische Höhe $S a$ und die mittlere quadratische Höhe $S q$ bei rein mechanischer Beanspruchung über der Versuchszeit nahezu konstant sind. Aufgrund der gewählten Belastung in Kombination mit der kurzen Versuchsdauer ist für den rein mechanisch belasteten Referenzversuch ein solcher Verlauf der Oberflächenparameter zu erwarten.

Durch die zusätzliche Beaufschlagung des Prüflings mit einer elektrischen Belastung kommt es zu einer Veränderung der $S a$ und $S q$ Werte hin zu einem über der Versuchszeit konstanten Level. Beide Kennwerte sinken unterhalb des Niveaus des mechanischen Referenzversuches. Dieses Verhalten zeigt sich unabhängig vom elektrischen Lastniveau. Ein klarer Zusammenhang zwischen der Höhe der anliegenden Lagerspannung und der sich einstellenden Oberflächenparameter ist bei dieser Art der Auswertung nicht ersichtlich.

Abb. 4 zeigt die Abbott-Firestone Kurven sowie Höhenkarten und Histogramme der Rauheiten der Oberflächen für die jeweiligen Versuche zum Ende der Versuchszeit (72h). Hierbei sei darauf hingewiesen, dass bei den visualisierten Oberflächen die Form der Laufbahn gefiltert wurde und die mikroskopische Aufnahme auf der Laufspur erfolgte. Weiterhin wurde zur besseren Vergleichbarkeit der Rauheiten die Bezugsebene so gewählt, dass sich der Mittelwert der Rauheiten zu Null ergibt. Weiterhin sind ergänzend zu den Oberflächenparametern in Abb. 3 in Tab. 2 die Oberflächenkennwerte Kernhöhe/Sk, die reduzierte Spitzenhöhe/Spk und die reduzierten Talhöhe/Svk aufgeführt. Mittels diese Oberflächenparameter können die Abbott Firestone Kurven der einzelnen Versuche in geeigneter Form miteinander verglichen werden.

Die Unterschiede in den Ausprägungen der Rauheiten zwischen den Versuchen sind klar ersichtlich. Während sich

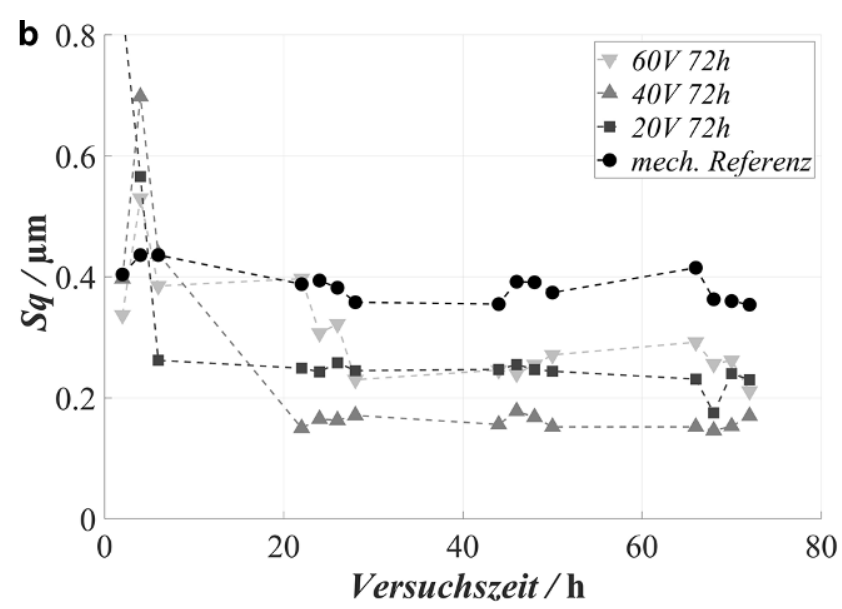

Abb. 3 Veränderung ausgewählter Oberflächenparameter über der Versuchszeit mit Hervorhebung der expliziten Auswertepunkte a Sa/mittlere arithmetische Höhe b Sq/mittlere quadratische Höhe 


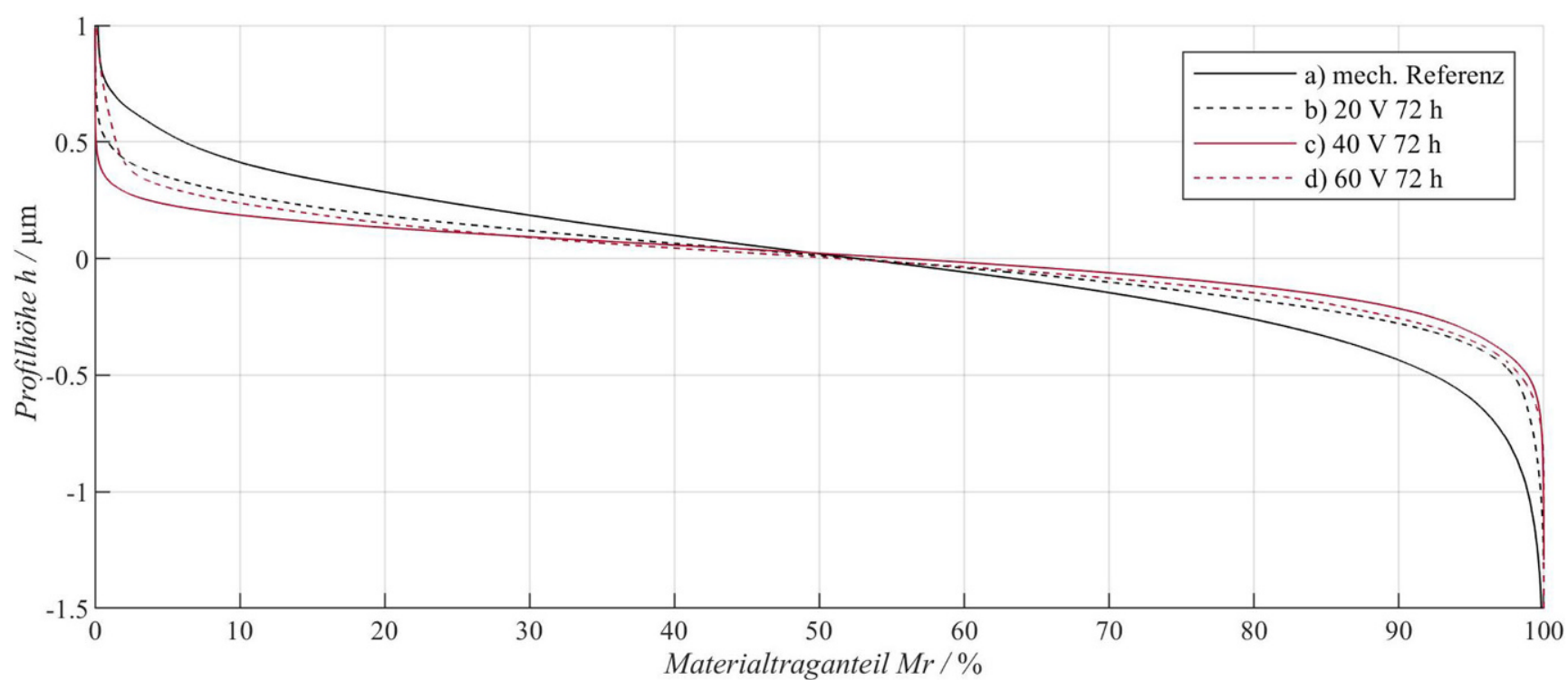

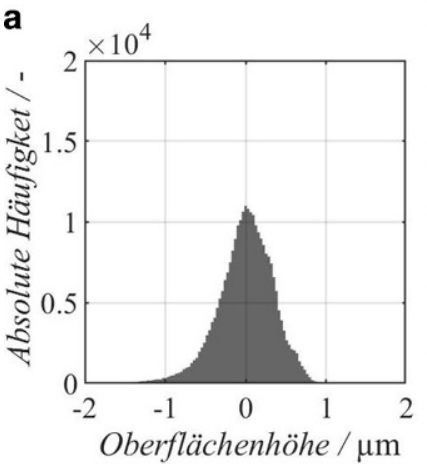

b
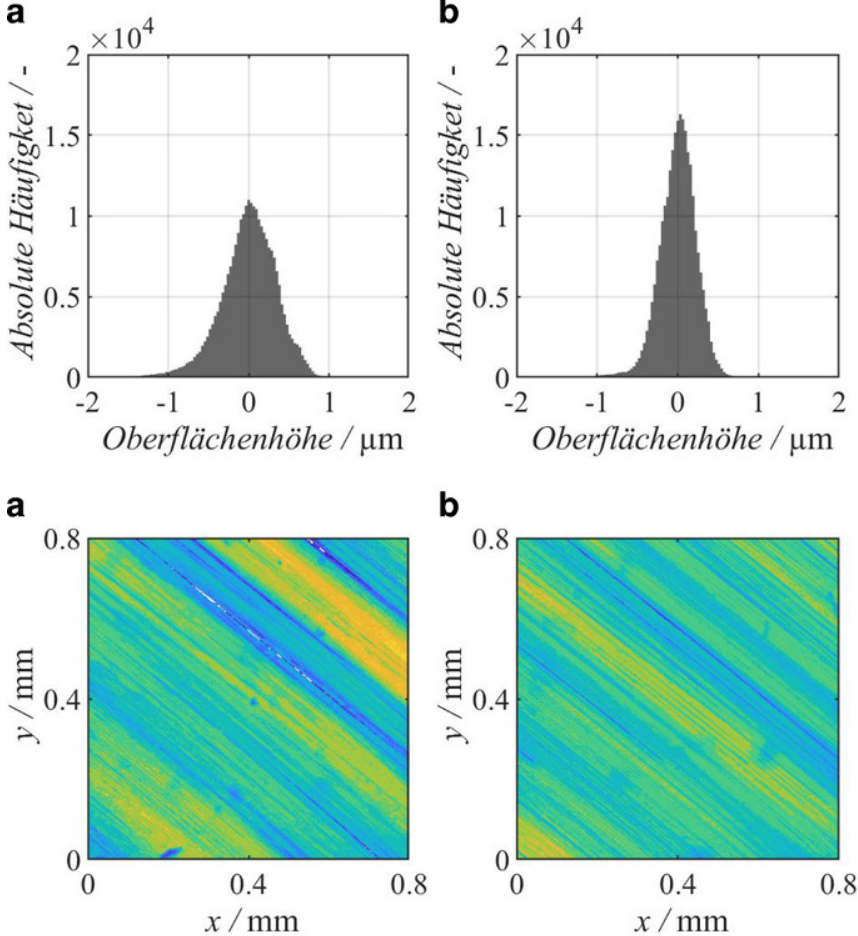

b

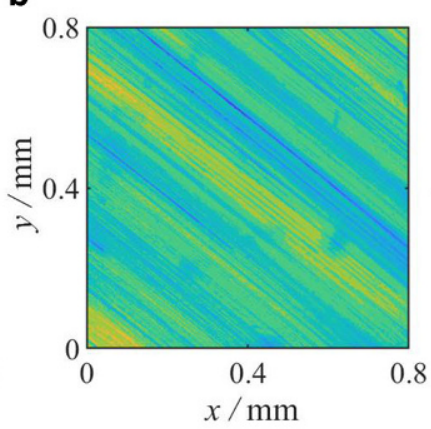

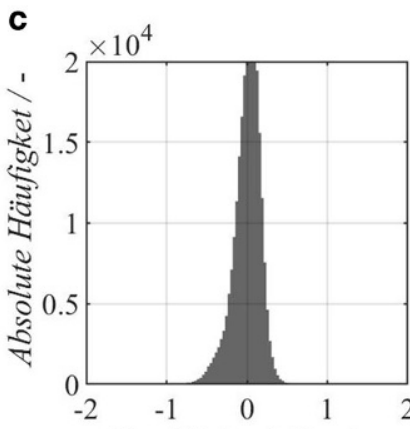

Oberflächenhöhe / $\mu \mathrm{m}$

c

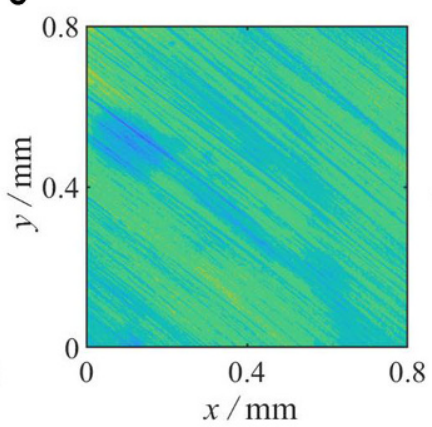

d

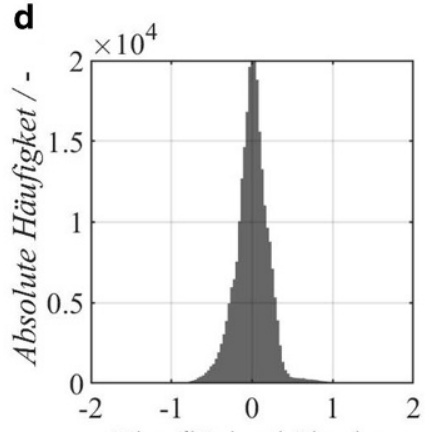

Oberflächenhöhe / $\mu \mathrm{m}$

d

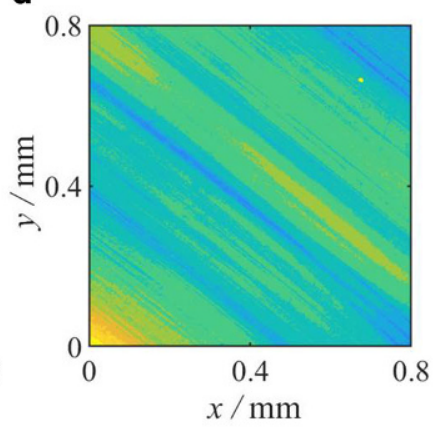

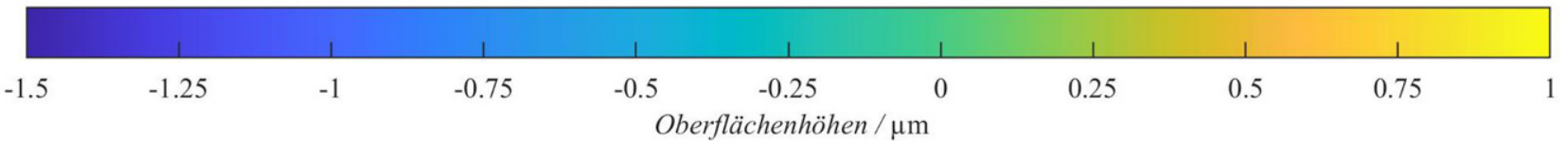

Abb. 4 Abbott-Firestone Kurven, Histogramme und Höhenkarten der Versuchsbauteile nach Abschluss der Versuchslaufzeit

die Höhenkarte im Vergleich zum Ausgangszustand [11] der mechanischen Belastung nur geringfügig ändert, werden bei den Versuchen mit zusätzlicher elektrischer Belastung die Oberflächenhöhen stark reduziert und die sich ausbildenden scheinbaren Kontaktflächen vergrößert. Diese Vergrößerung des Traganteils, ist bereits aus der Reduzierung der in Tab. 2 aufgeführten funktionalen Ober- flächenparameter ersichtlich. Eine weitere Klassifizierung der Traganteilssteigerung und die Herstellung des Zusammenhangs zwischen wirkender Last, der Deformation der Rauheiten und der sich einstellenden Kontaktfläche sind Gegenstand der Untersuchungen in Kap. 4. Darüber hinaus konnten bei diesen Versuchen in den überwachten Oberflächenausschnitten keine EDM typischen Krater entdeckt 
Tab. 2 Übersicht funktionaler Oberflächenparameter zur Beschreibung der AbbottFirestone Kurve

\begin{tabular}{llll}
\hline Versuch & \multicolumn{2}{l}{ Oberflächenparameter } \\
\cline { 2 - 4 } & Kernhöhe/Sk & Reduzierte Spitzenhöhe/Spk & Reduzierte Talhöhe/Svk \\
\hline a) mech. Referenz & 0,83 & 0,58 & 1,07 \\
b) 20V 72h & 0,55 & 0,43 & 0,89 \\
c) 40 V 72h & 0,37 & 0,39 & 0,56 \\
d) 60V 72h & 0,44 & 1,04 & 0,73 \\
\hline
\end{tabular}

werden. Dies spricht klar für einen Betrieb im tribologischen Zustand der Mischreibung bei den gewählten Randbedingungen [11].

\section{Eingangsgrößen zur Modellbildung}

Nachfolgend werden die für die Modellbildung und Modellierung notwendigen Eingangsgrößen aufgezeigt und dargelegt, wie diese ermittelt wurden. Hierbei wird zunächst die Vermessung der Oberflächen zur geometrischen Abbildung in der Simulation beschrieben, anschließend der entwickelte Modellaufbau erläutert sowie das zur Abbildung des nichtlinearen Materialverhaltens verwendete Modell dargestellt.

\subsection{Oberflächenvermessung}

Zur Vermessung der Wälzlagerlaufbahnoberflächen wurde ein Konfokal-Mikroskop der Firma $\mu$ surf verwendet. Im Gegensatz zu einem Lichtmikroskop, das ein Objekt möglichst gleichmäßig komplett ausleuchtet, wird die Beleuchtung an einem Konfokal-Mikroskop auf einen möglichst kleinen Beobachtungspunkt fokussiert [31].

Die Nachbearbeitung und Aufbereitung der Oberflächen erfolgt über das kommerzielle Programm MountainsMap ${ }^{\circledR}$ (ebenfalls von der Firma $\mu$ surf). In diesem Schritt wird zunächst nach so genannten Artefakten, wie Spiegelungen, Reflektionen oder starken Messabweichungen der Oberflächenmessung gesucht und mittels Interpolation beseitigt. Darauf aufbauend wird dann zwecks weiterführender Auswertung die Form von der Rauheit getrennt wodurch eine Ebene mit überlagerter Rauheit und Welligkeit entsteht.

Der vermessene Bereich der nachfolgend untersuchten Oberflächen betrug unter Verwendung eines Objektives mit zwanzigfacher Vergrößerung $800 \mu \mathrm{m} \times 800 \mu \mathrm{m}$ welche über $512 \times 512$ Datenpunkte abgebildet wurden. Als Eingangsgröße für das numerische Modell wurde ein repräsentativer Ausschnitt von $256 \times 256$ Datenpunkten je Oberfläche gewählt.

\subsection{Aufbau des numerischen Modells}

Nachfolgend wird der Aufbau des verwendeten Modells zur Simulation von Kontakten unter Berücksichtigung der rauen Oberfläche beschrieben. Für diese Problemstellung ist die hier verwendete Modellierungsstrategie mittels der CEL Methode bisher weitestgehend unbekannt. Wie in Kap. 1 beschrieben, vermeidet dieser Ansatz die Nachteile der klassischen Lagrangen FE die in diesem Simulationsumfeld auftreten können und stellt somit eine ernstzunehmende Alternative zu den auf diese Problemstellung angewendeten Simulationsmethoden dar.

Abb. 5 visualisiert durch eine exemplarische 2D Skizze den Aufbau des zur Abbildung des mechanischen rauen Kontaktes entwickelten numerischen Modells mittels der CEL Formulierung.

Die vermessene Oberfläche wird zunächst zu einem Volumenkörper mit definierter Dicke extrudiert. Hierdurch entsteht ein Quader mit einer rauen und fünf ebenen Mantelflächen, an welchen später die Randbedingungen definiert werden.

Nach der Überführung der rauen Oberfläche in einen Volumenkörper mit einer rauen Oberfläche und glatten Berandungen, wird dieser in ein Eulergitter integriert. Hierzu werden sowohl der Volumenkörper als auch der diskretisierte Eulerraum übereinander gelegt und das Volumen mit dazugehörenden Informationen (wie Materialbeschreibung, Füllgrad des Elementes) in die überlappenenden Eulerelemente eingeprägt. Mittels dieses Vorgangs erhält das Eulergitter zum einen die Information, in welchen Elementen „Volumen/ein physischer Körper“" vorhanden ist und zum anderen die Information, welches „Material“ in welcher Materialformulierung an dieser Stelle vorliegt. Dadurch, dass der Füllstand eines Elementes in der zur Simulation genutzten Finite Elemente Umgebung (ABAQUS CAE) nicht ausschließlich binär ( 0 oder $1 /$ leer oder voll), sondern auch Zwischenstufen annehmen kann, als auch dem Umstand, dass Elementfüllstände auf die Elementknoten approximiert werden, kann die Oberflächenrauheit inklusive Schrägungen abgebildet werden (vgl. [24]), welche dann wiederum bei der Kontaktfindung berücksichtig werden. Die Kontaktformulierung erfolgt ohne Berücksichtigung von Reibungseffekten. Ursächlich hierfür ist, dass in den generierten Simulationen primär Normalkräfte abgebildet werden und tangentiale Effekte vernachlässigt werden sollen.

Die Mantelflächen des Eulerraums werden entsprechend Abb. 5 mit Randbedingungen versehen, so dass ein Ausströmen von Material an den Rändern des Eulerraums nicht möglich ist. Weiterhin werden hier Stoßwellen nicht re- 
Abb. 5 Modellaufbau zur Simulation einer rauen Kontaktsituation mittels Euler-Formulierung der Starrkörperbewegungen

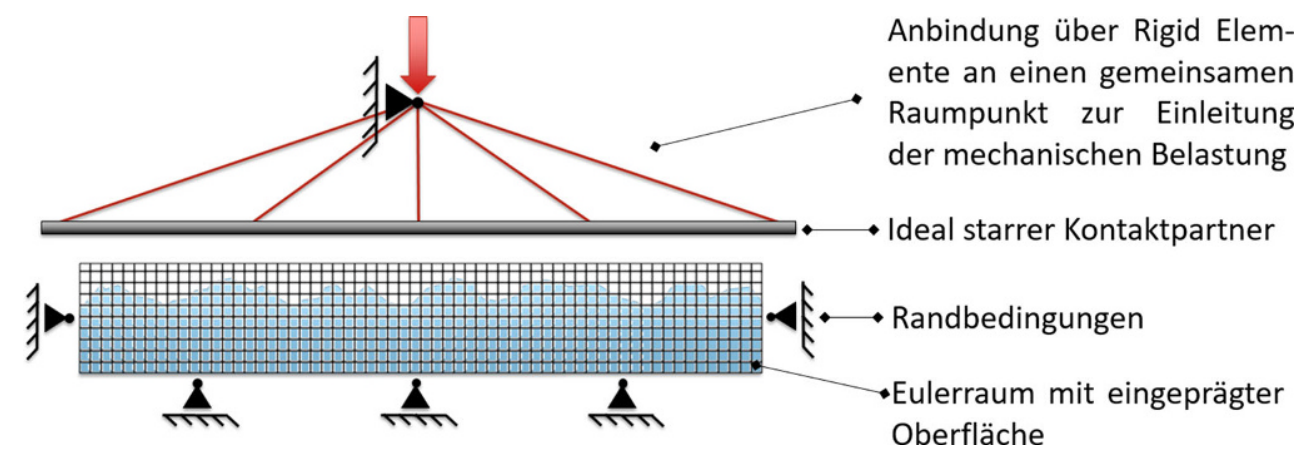

flektiert, sondern aus dem Modell ausgeleitet. Als Kontaktpartner für die raue Oberfläche wird ein ideal glattes und als ideal starr angenommenes Schalenelement in LagrangeFormulierung verwendet. Zur vereinfachten Implementierung der mechanischen Lastkomponente wird dieser Kontaktpartner über einen einzelnen Referenzpunkt gesteuert, mit dem er über ideal starre Stabelemente gekoppelt ist. Die Bewegung des Raumpunktes ist nur in Lastrichtung freigegeben, die tangentialen und rotatorischen Freiheitsgrade sind gesperrt.

Die mechanischen Lasten der Modelle orientieren sich hierbei an der Streckgrenze und Zugfestigkeit der verwendeten Materialien und führen zu einem maximalen nominellen Kontaktdruck von $2500 \mathrm{MPa}$ (entspricht einer Normalkraft von $400 \mathrm{~N}$ ).

Um die geringstmögliche Manipulation der Oberflächen zu gewährleisten wurde der Diskretisierungsabstand im Eulergitter so gewählt, dass ein aufgenommener Höhendatenpunkt der Oberfläche, auch einem Diskretisierungspunkt entspricht. Somit ergibt sich für den Eulerraum, als auch den Kontaktkörper konform zum Teilausschnitt der vermessenen Oberfläche eine Diskretisierung von $256 \times 256$ Elementen in der Ebene. Weiterhin werden entlang der Tiefenextrusion der Oberfläche 40 Elemente (entspricht $\sim 0,062 \mathrm{~mm}$ ) für den Eulerkörper vorgesehen (vergleiche Abb. 5).

Eine über diese Tiefe hinausgehende Berücksichtigung einer Struktursteifigkeit, basierend auf der makroskopischen Geometrie, wird in dieser Modellierungsausprägung nicht vorgesehen und wird an dieser Stelle vernachlässigt.

\subsection{Materialverhalten}

Zur Abbildung des nichtlinearen Materialverhaltens und einer damit einhergehenden exakteren Abschätzung der sich ausbildenden Spannungsspitzen und Teilplastifizierungen der rauen Oberflächen wird die Materialbeschreibung nach Johnson und Cook [32] verwendet. Hierbei handelt es sich um ein empirisches Modell basierend auf experimentell ermittelten Werkstoffkennwerten. Gemäß Gl. 1 setzt sich das Johnson-Cook Modell aus drei miteinander gekop- pelten Termen zusammen, einem zur Beschreibung der plastischen Dehnung, einem zur Klassifizierung des Einflusses der Dehnrate auf die Verfestigung des Materials als auch einem Term zur Berrücksichtigung von thermischen Entfestigungseffekten.

$\sigma_{F}=\left[\boldsymbol{A}+\boldsymbol{B} e_{p}{ }^{n}\right]\left[\mathbf{1}+\boldsymbol{C} \ln \frac{\dot{\boldsymbol{e}}_{p}}{\dot{\boldsymbol{e}}_{\mathbf{0}}}\right]\left[\mathbf{1}-\left(\frac{\boldsymbol{T}-T_{r}}{T_{m}-T_{r}}\right)^{m}\right]$

$\sigma_{\mathrm{F}} \quad$ Von Mises Fliessspannung

A Streckgrenze

B Verfestigungskoeffizient

$\mathrm{e}_{\mathrm{p}} \quad$ Plastische Vergleichsdehnung

$\mathrm{n}$ Verfestigungsexponent

C Dehnratenkoeffizient

$\dot{\boldsymbol{e}}_{p} \quad$ Plastische Vergleichsdehnrate

$\dot{\boldsymbol{e}}_{\mathbf{0}} \quad$ Anliegende Dehnrate

$\mathrm{T}$ Anliegende Temperatur

$\mathrm{T}_{\mathrm{r}} \quad$ Raumtemperatur

$\mathrm{T}_{\mathrm{m}} \quad$ Schmelztemperatur

m Thermischer Entfestigungsexponent

Sowohl eine weiterführende Beschreibung des Modells als auch der durchzuführenden Versuche zur Ermittlung der Werkstoffparameter, können neben [32] auch [33] entnommen werden. Für die Simulationen ist der Plastifizierungsterm:

$\sigma_{F}=\left[\boldsymbol{A}+\boldsymbol{B} e_{p}{ }^{n}\right]$

wesentlich, da die durchgeführten Betrachtungen für $\mathrm{T}=\mathrm{T}_{\mathrm{r}}$ gelten und kinetische Effekte $\left(\dot{e}_{p}=\dot{e}_{0}\right)$ auf die Rauheit zunächst nicht untersucht werden.

Die in der Modellierung verwendeten Materialparameter für einen $100 \mathrm{Cr} 6$ Wälzlagerstahl, können Tab. 3 entnommen werden. Diese Modellparameter stammen aus [34] und wurden an Probekörpern mit ähnlicher Vergütungsstufe ermittelt, weswegen Sie hier ebenfalls verwendet werden können. Diese Parameter zeigten in diversen weiteren Veröffentlichungen und Referenzierungen (u. a: $[33,35]$ ). ihre 


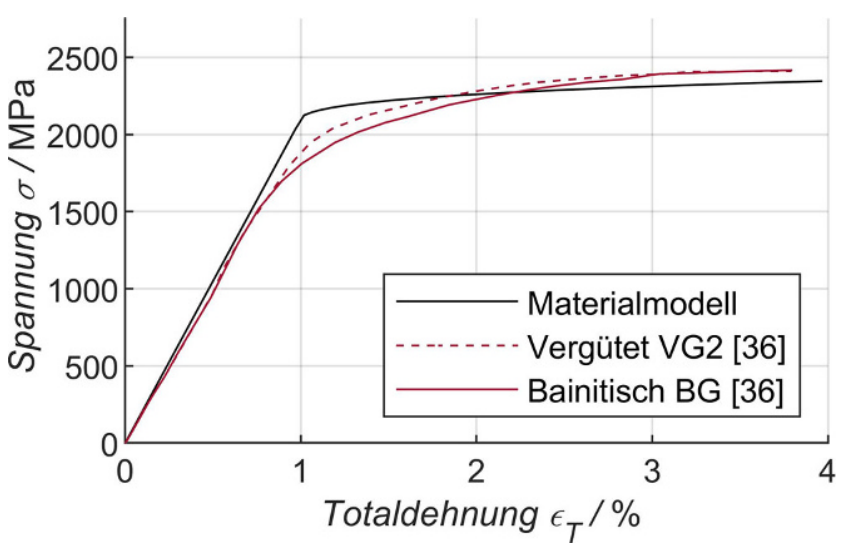

Abb. 6 Gegenüberstellung der Spannungs-Dehnungsbeziehung des verwendeten Materialmodells mit Zugkurven vergleichbarer Vergütungsstufe aus [36]

Tab. 3 Kennwerte für das verwendete Materialmodell

\begin{tabular}{lll}
\hline $\mathrm{A} / \mathrm{MPa}$ & $\mathrm{B} / \mathrm{MPa}$ & $n /-$ \\
\hline 2033 & 895 & 0,3 \\
\hline
\end{tabular}

Gültigkeit. Weiterhin wird in Abb. 6 ein Abgleich zwischen experimentell ermittelten Zugkurven mit vergleichbar vergüteter Werkstoffqualität und dem Materialmodell, unter Verwendung der Parameter gemäß Tab. 2, dargestellt.

\section{Ergebnisse}

Aufbauend auf den in Abb. 4 dargestellten Abott Firestone Kurven werden nachfolgend die Auswirkungen der Glättung auf die Deformations-/Kraftbeziehung der Oberflächen dargestellt. Hierzu werden die Ergebnisse der Simulationen der vier experimentell erzeugten Oberflächen in drei Teilbereichen analysiert. Zunächst wird der Einfluss der zusätzlich wirkenden elektrischen Belastung auf den Traganteil dargestellt. Hierauf aufbauend werden dann Unterschiede in der Ausbildung der plastischen Dehnung unter den Oberflächen bei einer reinen mechanischen Belastung dargestellt. Abschließend werden dann Spannungsverteilungen unter den Oberflächen miteinander verglichen und einander gegenübergestellt.

\subsection{Traganteilssteigerung}

Die simulierten Traganteilskurven der sich nach den Experimenten eingestellten Oberflächen (kombinierter elektrischer und mechanischer Belastung zu Ergebnissen der mechanischen Referenzoberfläche) sind in Abb. 7 einander gegenübergestellt. Basierend hierauf erfolgt die weiterführende Diskussion und Interpretation der Ergebnisse. Der Abbildungsteil a visualisiert den Verlauf des Tragan- teils, definiert als das Verhältnis der lokalen zur nominellen Kontaktfläche, über dem nominellen Kontaktdruck. Der nominelle Kontaktdruck wird definiert als der Quotient aus der im Berechnungsschritt aufgeprägten Kraft und der nominellen Kontaktfläche (vgl. Kap. 1). Im Abbildungsteil b ist für die durchgeführten Oberflächensimulationen der plastifizierte Materialanteil des Modells dargestellt. Dieser errechnet sich aus dem Verhältnis der Anzahl der Elemente, welche eine plastische Deformation erfahren haben, bezogen auf die Gesamtanzahl der Elemente des Modells in welchen zum jeweiligen Berechnungszeitpunkt die Materialinformation des verwendeten Werkstoffes vorlag. Starke lokale Plastifizierungen an den Rauheitsspitzen, wie sie gerade bei moderaten Kontaktdrücken $(<1000 \mathrm{MPa}) \mathrm{zu}$ erwarten sind, können in dieser Form der Auswertung nur unvollständig dargestellt werden.

Augenscheinlich ist, dass die Oberflächen mit zusätzlicher elektrischer Belastung sich so stark eingegelättet haben, dass es bereits bei sehr niedrigen Kontaktdrücken zu hohen Flächentraganteilen kommt (vgl. Abb. 7a). Weiterhin wird hier verdeutlicht, dass in Folge der niedrigeren Rauheiten die Zunahme des Flächentraganteils über dem steigenden nominellen Kontaktdruck wesentlich geringer ausgeprägt ist, als etwa die der Referenzoberfläche.

Um die Auswirkungen der nichtlinearen Materialbeschreibung mit zunehmender Simulationszeit respektive der Kontaktdrucksteigerung zu verdeutlichen, kann der plastifizierte Materialanteil (Abb. 7b) in den Modellen miteinander verglichen werden. So bestätigt $\mathrm{Abb}$. $7 \mathrm{~b}$, dass die deutlich glatteren elektrisch beanspruchten Oberflächen bei einer äquivalenten äußeren Belastung einen deutlich geringeren plastifizierten Materialanteil aufweisen. Weiterhin zeigt der Verlauf dieses Parameters bei der mechanischen Referenzkurve einen ausgeprägten nichtlinearen Charakter. So kommt es bei einer Überschreitung des nominellen Kontaktdrucks von etwa $\sim 2200 \mathrm{MPa}$ zu einem sprunghaften Anstieg des plastifizierten Materialanteils. Ursächlich hierfür ist das auf den Oberflächenrauheiten basierende inhomogene Spannungsfeld (siehe Abschn. 4.3). So wird ab diesem Zeitpunkt an mehreren Stellen die Fließgrenze überschritten und es kommt zu einem großflächigen Fließen, um die applizierte Beanspruchung durch Plastifizierung mit einhergehender Steigerung der Festigkeit zu ertragen.

\subsection{Plastifizierung der Modelloberflächen}

Zum besseren Vergleich und Bewertung der vorliegenden Plastifizierung und ihrem Einfluss in den jeweiligen Modellen dient zusätzlich zu Abb. 7b Abb. 9. Während in Abb. 7b der dreidimensionale Charakter der Plastifizierung in einen 1D Kennwert überführt wird, soll Abb. 9 eine Übersicht über die Ausbreitung und die Höhe der Plastifizierung un- 
a

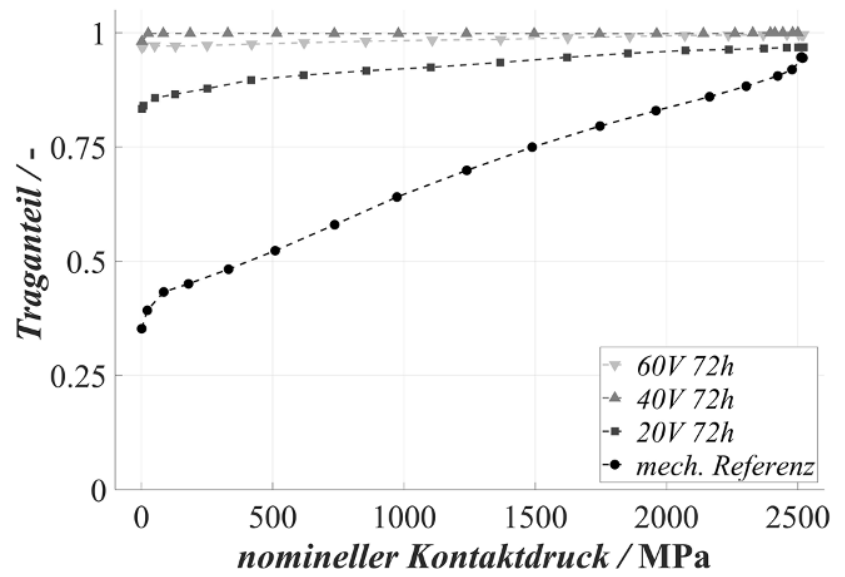

b

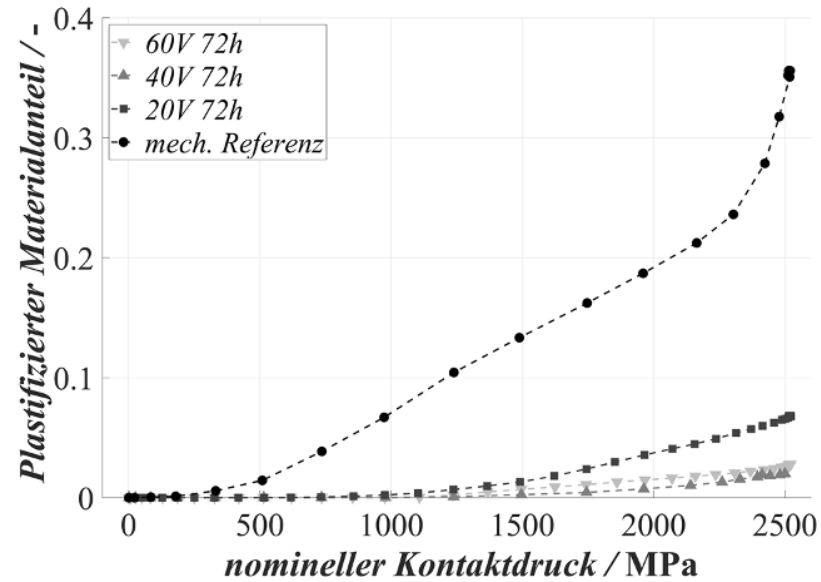

Abb. 7 Auswertung der Simulationsergebnisse der untersuchten Oberflächen über dem nominellen Kontaktdruck mittels dem Traganteil (a) und dem plastifizierten Materialanteil (b)

ter der Oberfläche in Abhängigkeit des nominellen Kontaktdrucks liefern. Hierbei wird nur der Bereich unter der Oberfläche visualisiert, bis zu dem Plastifizierungseffekte im Modell auftreten.

Als Maß für die Plastifizierung wird neben der gemittelten plastischen Dehnung auch der Parameter der gemittelten Fließgrenze eingeführt. Die Fließgrenze beschreibt im Spannungsdehnungsdiagramm eines vorzugsweise metallischen Werkstoffes den Punkt, in dem das elastische Materialverhalten, definiert durch die Hook'sche Gerade, in ein plastisches Verhalten übergeht. Mit fortschreitender Plastifizierung steigt die Fließgrenze an und kann somit, neben der plastischen Dehnung, als Maß für die im Modell vorliegende Plastizität verwendet werden. Da beide Werte jeweils über das verwendete Materialgesetz ineinander umgerechnet werden können, gibt die Legende in Abb. 9 auch Auskunft über beide Parameter.

Die dargestellte Mittelung dieser Kennwerte erfolgt aus den Ergebnissen für jeden Berechnungsschritt und abhängig von der Materialtiefe. Abb. 8 zeigt visuell und durch Formeln ergänzt, wie diese Mittelung in jedem Berechnungsschritt vorgenommen wird. Hierzu werden zum jeweiligen Auswertezeitpunkt die plastischen Dehnungen aus dem Modell extrahiert. Darauf aufbauend wird dann eine Mittelung in der x-y Ebene für jede Tiefenkoordinate z entlang des Berechnungsgitters durchgeführt (vgl. Gl. 3). Auf der Basis der so bestimmten mittleren plastischen Dehnung wird dann über den formalen Zusammenhang in Gl. 4 die mittlere Fließgrenze für den betrachteten Simulationszeitpunkt (korrespondierend zum nominellen Kontaktdruck) berechnet. Diese gemittelte plastische Dehnung und Fließgrenze wird dann abhängig von der Oberflächenkoordinate (Materialtiefe) über dem nominellen Kontaktdruck aufgetragen, wodurch die Höhenliniendarstellung in Abb. 9 entsteht.
Für $k=0$ nach l ergibt sich die mittlere plastische Dehnung zu:

$\bar{\varepsilon}_{P}(\mathrm{k})=\frac{\sum_{\mathrm{i}=0}^{\mathrm{m}} \sum_{\mathrm{j}=0}^{\mathrm{n}} \varepsilon_{P}(\mathrm{i}, \mathrm{j}, \mathrm{k})}{\mathrm{mxn}}$

Sowie die mittlere Fließgrenze:

$\bar{\sigma}_{v}(\mathrm{k})=\left[A+B \bar{\varepsilon}_{P}(\mathrm{k})^{n}\right]$

Die in Abb. 9 zusammengefassten Ergebnisse zeigen eine deutliche Auswirkung der Rauheiten auf die sich einstellende Plastifizierung unter der Oberfläche. Ebenso ist ersichtlich, dass die durch Stromdurchgang geglätteten Oberflächen erst bei deutlich höheren Kontaktdrücken plastisch deformiert werden (mech. Referen $\rightarrow 100 \mathrm{MPa} / 20 \mathrm{~V}$ $72 \mathrm{~h} \rightarrow 600 \mathrm{MPa} / 40 \mathrm{~V} \quad 72 \mathrm{~h} \rightarrow 1000 \mathrm{MPa} / 60 \mathrm{~V} \quad 72 \mathrm{~h} \rightarrow$ $700 \mathrm{MPa}$ ). Ebenfalls unterscheidet sich auch der Bereich unter der Oberfläche bis zu dem eine Plastifizierung des Materials feststellbar ist (mech. Referenz $\rightarrow-0,03 \mathrm{~mm} /$ elektrische Simulation $\rightarrow-0,015 \mathrm{~mm}$ ).

An dieser Stelle sei angemerkt, dass die Oberflächenausschnitte bis zu einer Materialtiefe von etwa $0,062 \mathrm{~mm}$ modelliert wurden. Aus Abb. 9 ist erkennbar, dass eine Plastifizierung bis in eine Tiefe von $0,03 \mathrm{~mm}$ festgestellt werden konnte. In Folge dessen kann davon ausgegangen werden, dass ausreichend elastisches Grundmaterial modelliert wurde um Wechselwirkungen zwischen der auftretenden Plastifizierung und der Verschiebungsrandbedingung am Boden des Modells (x-y Ebene konform zu Abb. 8) auszuschlieBen.

Ein in Kauf genommener Nachteil der hier verwendeten Darstellung ist, dass gerade anfängliche lokale Plastifizierungen der höchsten Rauheitsspitzen durch die Mittelung nicht hervorgehoben werden. Mittels der Darstellung als 3D Plot oder durch mehrere Stirnschnitte in der $\mathrm{x}-\mathrm{z}$ Ebene 


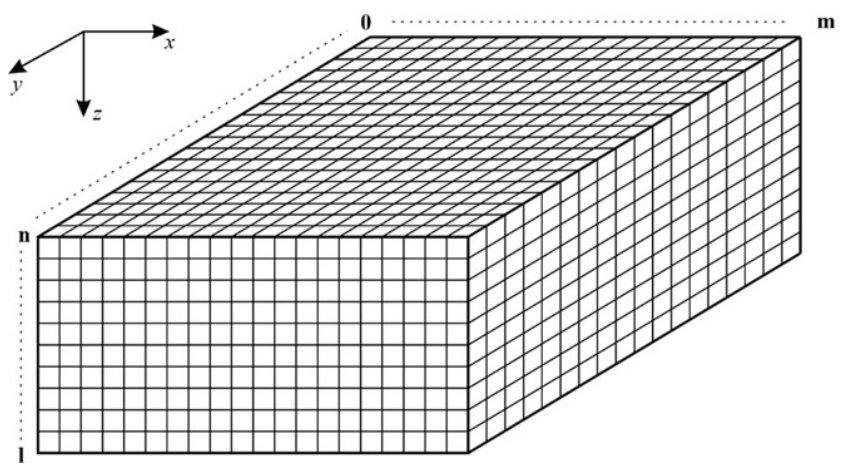

Abb. 8 Visualisierung des eulerschen Berechnungsraums zur Verdeutlichung der Berechnung der mittleren Plastifizierung und der mittleren Fließgrenze je Berechnungsschritt

könnten diese Plastifizierungen lokal aufgelöst dargestellt werden. Jedoch ist die detaillierte Analyse der Plastizität der Rauheitsspitzen nicht im Fokus der hier durchgeführten Auswertungen.

\subsection{Spannungsverteilung unter der Oberfläche}

Die Auswirkung der Rauheiten und des Einglättens infolge der elektrischen Belastung auf das Ausbilden des dreidimensionalen Spannungsverlaufs unter der Oberfläche werden in Abb. 10 verdeutlicht. Hierin wird die inhomogene Verteilung der von Mises Spannung in unterschiedlich tiefen Schnittebenen (x-y Ebene konform zu Abb. 8) visualisiert und für die vermessenen Oberflächen einander gegenübergestellt. Diese Auswertung erfolgt für eine Kontaktkraft von $400 \mathrm{~N}$, was einem nominellen Kontaktdruck von $2500 \mathrm{MPa}$ entspricht.

In der Auswertung der Spannungen (Abb. 10), für die Oberfläche aus dem rein mechanischen Referenzversuch, zeigt sich eine inhomogene Verteilung der von Mises Spannung bis hin zur Modellgrenze von $0,062 \mathrm{~mm}$. Hierbei können die Bearbeitungsspuren der Wälzlagerlaufbahn, in Form der Rauheiten, noch deutlich erkannt werden und Spannungsüberhöhungen festgestellt werden. Diese Inhomogenität ist bei den experimentell erzeugten Oberflächen, mit zusätzlicher elektrischer Belastung und einem Betrieb in Mischreibung, deutlich reduziert. So sind bei der Oberfläche $20 \mathrm{~V} 72 \mathrm{~h}$ im Spannungsverlauf noch einzelne Inhomogenitäten erkennbar. Jedoch sind diese im Gegensatz zur Oberfläche aus dem Referenzversuch deutlich weniger ausgeprägt. Die Simulationen der Oberflächen $40 \mathrm{~V} 72 \mathrm{~h}$ und $60 \mathrm{~V} 72 \mathrm{~h}$ zeigen knapp unterhalb der Kontaktebene (Materialtiefe $-0,0075 \mathrm{~mm}$ ) einzelne wenige lokale Spannungsspitzen. Diese werden zunehmend unterhalb der Oberfläche abgebaut und es bildet sich bei diesen Oberflächen, ab einer Materialtiefe von etwa $-0,03 \mathrm{~mm}$, ein nahezu homogenes Spannungsbild aus. Inwieweit diese Hotspots auf reale Rauheiten zurückzuführen sind oder ob es sich um geringe Messabweichungen beim Vermessen der Oberfläche handelt, welche dann zu den dargestellten Spannungsspitzen führen, kann nicht abschließend festgestellt werden.

\subsection{Zusammenführung der Simulationsergebnisse}

In den Ergebnissen konnte gezeigt werden, dass sich der initiale Traganteil in Folge der zuvor anliegenden elektrischen Belastung signifikant erhöht, sich jedoch die Unterschiede zur rein mechanischen Oberfläche mit zunehmenden Belastungen und damit steigendem nominellen Kontaktdruck reduzieren. Ursächlich hierfür ist die fortschreitende Plastifizierung der einzelnen Rauheitsspitzen und dem damit verbundenen mechanischen Glätten der Rauheiten. Weiterhin zeigen auch die ausgewerteten Kurven von ihrem Verlauf ein unterschiedliches Verhalten. Während die Traganteilskurve der Referenzoberfläche einen nichtlinearen Verlauf über dem Kontaktdruck zeigt, sind die Kurven der elektrisch belasteten Oberflächen, im Vergleich, nahezu linear. Dieses Verhalten lässt sich aufgrund der nicht stark ausgeprägten Rauheiten und einer damit verbundenen Reduzierung der Plastifizierung erklären. Besonders deutlich zeigt sich diese Einglättung bei den elektrischen Lastleveln von 40 und $60 \mathrm{~V}$ was einer gemessenen Lagerspannung von 6,6 und $10 \mathrm{~V}$ entspricht. Die Traganteilskurven dieser beiden Lastfälle unterscheiden sich trotz der unterschiedlichen elektrischen Belastung nur marginal voneinander. Basierend auf der Auswertung der Bauteilspannungen dieser beiden Lastfälle (vgl. Abb. 9 und 10), kann jedoch davon ausgegangen werden, dass die leicht unterschiedlichen Oberflächenstrukturen die Ursache für dieses Verhalten sind. Die Traganteilssimulation der mit $20 \mathrm{~V}$ Zwischenkreisspannung belasteten Oberfläche zeigt ebenfalls eine deutliche Traganteilssteigerung im Vergleich zur Referenzoberfläche.

Als Auswirkung der hier entstandenen Einglättung bei den elektrisch belasteten Proben bildet sich zudem ein deutlich homogenerer Spannungsverlauf unter den Oberflächen aus (Abb. 10). Hierdurch kommt es auch zu geringeren Plastifizierungen im Material (Abb. 7b). Diese ist nicht nur nach dem absoluten Wert niedriger, sondern auch hinsichtlich ihrer Ausbreitung unter der Oberfläche (Abb. 9) deutlich reduziert.

Basierend auf den Abott Firestone Kurven (Abb. 4 und Tab. 2) ist bereits eine erste Aussage möglich wie sich die Oberflächenstrukturen unter Last verhalten werden. Zur detaillierten Analyse der mechanischen Auswirkungen der Oberflächenstruktur dienen jedoch die Ergebnisse der CEL Kontaktsimulation. So liegt etwa bei der Betrachtung der Abbott Firestone Kurven die Vermutung nahe, dass sich die Traganteilskurven von $20 \mathrm{~V} 72 \mathrm{~h} \mathrm{zu} 60 \mathrm{~V} 72 \mathrm{~h}$ nur geringfügig voneinander unterscheiden. Im Kontext von Abb. 7a zeigt sich jedoch bei niedrigen Lasten ein signifi- 
Abb. 9 Gemittelte Fließgrenze und gemittelte plastische Dehnung unter der Oberfläche des Kontaktes in Abhängigkeit des nominellen Kontaktdrucks
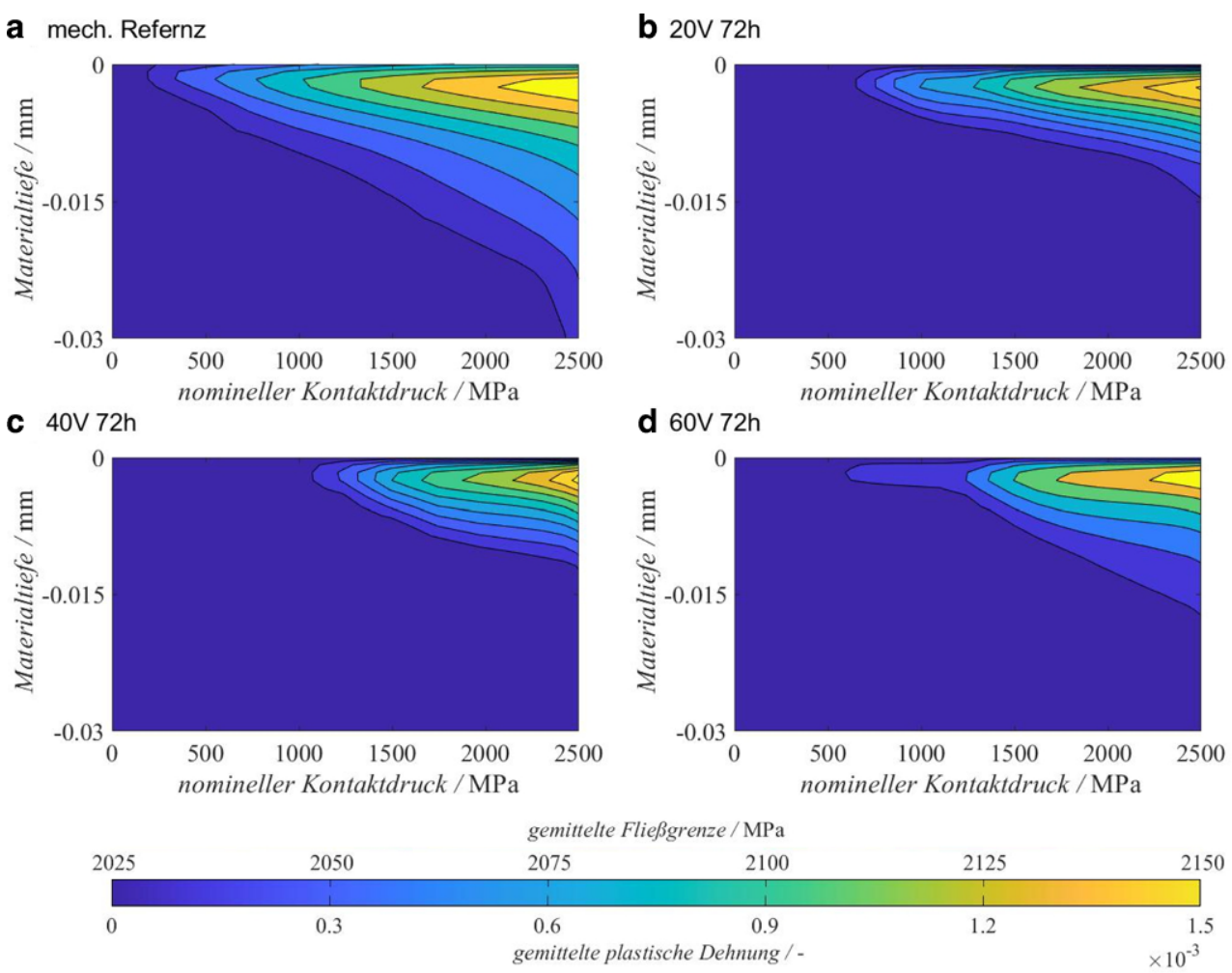

kanter Unterschied im Tragverhalten. So ist die Oberfläche des Versuchs $60 \mathrm{~V} 72 \mathrm{~h}$ bereits so stark eingeglättet, dass es bereits bei niedrigen Kontaktdrücken zu einem Traganteil nahe 1,0 kommt. Die Oberfläche $20 \mathrm{~V} 72 \mathrm{~h}$ hingegen weißt noch in dem Maße eine Rauheit auf, dass der Traganteil mit zunehmendem Kontaktdruck von etwa 0,8 auf 0,95 steigt. Die Differenzen in den Kernhöhen/Sk $(20 \mathrm{~V} 72 \mathrm{~h}$ Sk 0,55 zu 60V $72 \mathrm{~h} S k 0,44)$ der beiden Oberflächen deuten ebenfalls auf dieses unterschiedliche Tragverhalten hin, welches in der Simulation bestätigt wurde. Hierauf aufbauend zeigt die Simulation die Auswirkungen in der mechanischen Beanspruchung der Oberflächen. So kommt es bei der Oberfläche $20 \mathrm{~V} 72 \mathrm{~h} \mathrm{zu}$ einer ausgeprägten Plastifizierung bereits bei niedrigeren Kontaktdrücken, als auch einer inhomogeneren Spannungsverteilung unter der Oberfläche im Vergleich zu der Oberfläche 60 V $72 \mathrm{~h}$.

Die zu erwartenden Unterschiede in den Traganteilen der $40 \mathrm{~V} 72 \mathrm{~h} \mathrm{zu} 60 \mathrm{~V} 72 \mathrm{~h}$ auf Basis der unterschiedlichen Abbott Kurven konnte die Simulation nicht bestätigen. Beide Oberflächenstrukturen sind bereits so stark geglättet, dass die Traganteile bei einem niedrigen Kontaktdruck nur noch marginal voneinander abweichen. Ein Indiz hierfür stellte bereits die vergleichbaren Kernhöhen der beiden Oberflächen (40 V 72 h $S k 0,37$ zu $60 \mathrm{~V} 72$ h $S k$ 0,44) da. Unabhängig hiervon zeigen sich auch hier Unterschiede in den mechanischen Auswirkungen in Folge der anliegenden Belastung. So weisen die Oberflächen eine anders geartete Ausbreitung der gemittelten plastischen Dehnung, als auch Un- terschiede in der Homogenität der Spannungsausbreitung unter der Oberfläche auf. Eine mögliche Ursache hierfür ist in den Unterschieden in der reduzierten Spitzenhöhe/Spk der beiden Oberflächen zu suchen. So weist die Oberfläche $40 \mathrm{~V} 72 \mathrm{~h}$ mit 0,39 eine deutlich niedrigere reduzierte Spitzenhöhe/Spk, als die Oberfläche $60 \mathrm{~V} 72 \mathrm{~h}$ mit 1,04 auf. In Folge dessen kommt es bei der Oberfläche $60 \mathrm{~V} 72 \mathrm{~h}$ in der Simulation zu einem gehäuften Kontakt einzelner Rauheitsspitzen und somit einer Ausbreitung einzelner punktuell höherbeanspruchten Zonen (vgl. Abb. 10/60 V 72 h) mit einhergehender Plastifizierung.

\section{Zusammenfassung und Ausblick}

In den vorliegenden Untersuchungen sind die Auswirkungen von Oberflächenglättungen, infolge von parasitärem elektrischem Stromdurchgang in einem Axiallager bei einem Betrieb in Mischreibung dargestellt. Die Beurteilung erfolgt mittels der Gegenüberstellung der Ergebnisse aus einer Kontaktsimulation unter Berücksichtigung der sich experimentell einstellenden mikroskopischen Oberflächenrauheiten. Hierzu wurden Traganteilskurven (Abschn. 4.1) simuliert sowie die sich einstellende Plastifizierung (Abschn. 4.2) und die inhomogene Spannungsausbreitung (Abschn. 4.3) unter der Oberfläche analysiert.

Zur Abbildung des Kontaktes kam ein Finite Elemente Ansatz auf Basis der kombinierten Euler-Lagrange-Metho- 

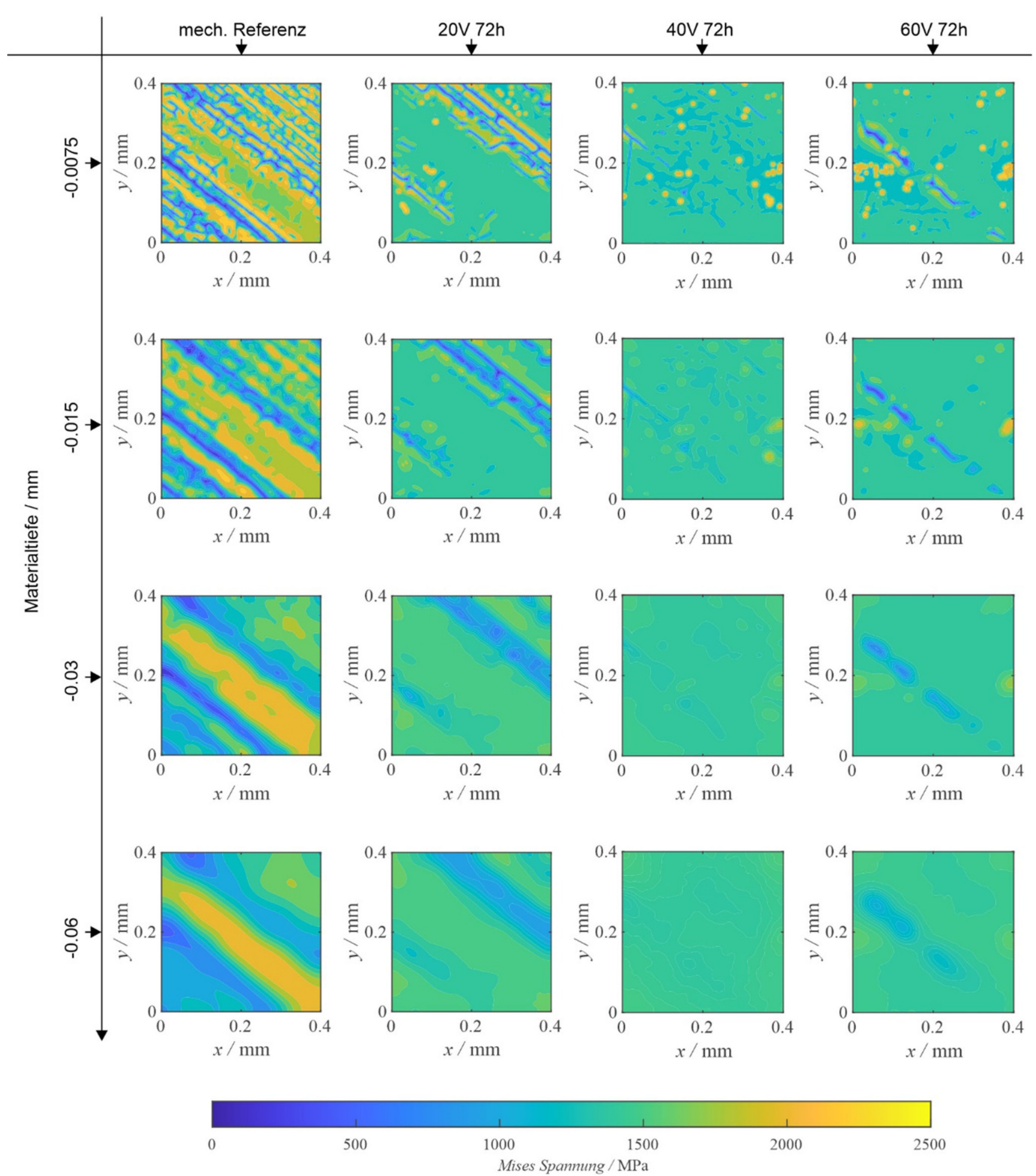

Abb. 10 Ausprägung der von Mises Vergleichsspannung abhängig von der simulierten Oberfläche in unterschiedlichen Schnittebenen unterhalb der Berührzone bei einem nominellen Kontaktdruck von $2500 \mathrm{MPa}$ 
de zum Einsatz (Kap. 3). Die Rauheiten wurden hierbei in ein Eulergitter eingeprägt und der Kontaktkörper als ideal glatter Lagrange-Körper modelliert (vgl. Abb. 3). Die Abbildung der Plastizität im CEL Modelle erfolgte mittels der Implementierung des Materialmodells nach Johnson und Cook. Somit erlaubt das hier entwickelte Modell die Untersuchung der Ausprägung der mechanisch induzierten Plastifizierung sowie die Spannungsverteilung unter der Oberfläche für die untersuchten Modellfälle. Die aus anderen Anwendungen bekannte und bewährte CEL Methode wurde bisher nicht auf Problemstellungen im Kontext der Simulation rauer Oberflächen angewendet und ergänzt in diesem Bereich die bestehenden Simulationswerkzeuge (Halbraummodelle, Lagrange FE Ansätze, zelluläre Automaten).

Als Eingangsgröße für diese entwickelte Simulation dienten drei experimentell erzeugte Oberflächen, welche mit einer kombinierten elektrischen und mechanischen Belastung beaufschlagt wurden. Zur Verdeutlichung des Einflusses der elektrischen Belastung bot sich an eine Oberfläche rein mechanisch zu belasten, um einen Vergleichsfall, im Gegensatz zu den mechanisch elektrischen Oberflächen, zu erhalten. Diese vier experimentell erzeugten Oberflächen wurden in das beschriebene numerische Modell überführt. Dieses Vorgehen erlaubt die getrennte Betrachtung der mechanisch induzierten Überrollglättung im Gegensatz zur unerwünschten elektrischen Glättung in Folge von Stromdurchgang.

Bei der hier quantifizierten elektrischen Glättung (Kap. 4) der Wälzoberflächen handelt es sich um einen Nebeneffekt des Phänomens des elektrischen Stromdurchgangs in Wälzlagern, welches bei einem Betrieb der Lagerung in der Mischreibung beobachtet werden kann. Ursächlich für diese Glättungen ist die zusätzliche eingeprägte elektrische Belastung in den Wälzkontakt mit der damit einhergehenden thermischen Belastung der Kontaktstelle. Vor diesem Hintergrund ist diese Glättung nicht zwingend nur auf Basis ihrer positiven Auswirkungen, wie:

- Tribologisch: früherer Übergang in die Vollschmierung und Steigerung des Traganteils;

- Mechanisch: Reduzierung der Rauheit und geringere Plastifizierung sowie eine homogenere Spannungsverteilung

zu beurteilen. Sondern muss auf der Basis der zusätzlich wirkenden elektrischen Belastung und deren Folgen interpretiert werden. So führt die hier beschriebene Änderung der Rauheit nicht nur zu einem veränderten tribologischen Verhalten, sondern auch zu einer Veränderung des elektrischen Ersatzsystems (vgl. Abb. 1). Damit einher geht auch ein Wechsel im Schadensmechanismus, weg vom ohm'schen Stromfluss über die sich berührenden Rauheitsspitzen, hin zu hochenergetischen Entladeströmen zwischen Wälzkörper und Wälzlagerlaufbahn. Weiterhin gilt es zu berücksichtigen, dass der parasitäre Stromfluss auch über den sich zwischen den Wälzoberflächen befindliche Schmierstoff erfolgt. Die gezeigte Oberflächenveränderung lässt somit auch Rückschlüsse auf die Belastung des Schmierstoffes zu.

Die hier gezeigten Ergebnisse liefern demnach nicht nur die Einebnung und die mechanischen Folgen des Stromdurchganges auf die Lagerlaufbahn, sondern können auch als Eingangsgrößen für weiterführende tribologische Simulationen dienen. Hieraus kann dann wiederum das elektrische Verhalten des Wälzlagers in Form von Impedanzsimulationen abgeleitet werden. Somit liefern die hier gezeigten Traganteilskurven einen weiteren Schritt, hin zu einer Auslegung von Wälzlagern unter kombinierter mechanischer und elektrischer Belastung.

Danksagung Diese Arbeit entstand im Rahmen der durch die Deutsche Forschungsgemeinschaft (DFG) e. V. finanziell geförderten Projekte „Modell zur Bestimmung der thermischen Beanspruchung von Schmierstoffen in Folge von mechanischen und elektrischen Belastungen im Wälzkontakt“ (Projektnr. 407468812) sowie „Bauteiloberflächen: Morphologie auf der Mikroskala“ (Projektnr. 172116086 - SFB 926).

Ein besonderer Dank gilt weiterhin dem Institut für elektrische Energiewandlung der TU Darmstadt, als auch der Firma Digital Surf für die Bereitstellung der Software MountainsMap ${ }^{\circledR}$.

Funding Open Access funding enabled and organized by Projekt DEAL.

Open Access Dieser Artikel wird unter der Creative Commons Namensnennung 4.0 International Lizenz veröffentlicht, welche die Nutzung, Vervielfältigung, Bearbeitung, Verbreitung und Wiedergabe in jeglichem Medium und Format erlaubt, sofern Sie den/die ursprünglichen Autor(en) und die Quelle ordnungsgemäß nennen, einen Link zur Creative Commons Lizenz beifügen und angeben, ob Änderungen vorgenommen wurden.

Die in diesem Artikel enthaltenen Bilder und sonstiges Drittmaterial unterliegen ebenfalls der genannten Creative Commons Lizenz, sofern sich aus der Abbildungslegende nichts anderes ergibt. Sofern das betreffende Material nicht unter der genannten Creative Commons Lizenz steht und die betreffende Handlung nicht nach gesetzlichen Vorschriften erlaubt ist, ist für die oben aufgeführten Weiterverwendungen des Materials die Einwilligung des jeweiligen Rechteinhabers einzuholen.

Weitere Details zur Lizenz entnehmen Sie bitte der Lizenzinformation auf http://creativecommons.org/licenses/by/4.0/deed.de.

\section{Literatur}

1. Punga F, Hess W (1907) Eine Erscheinung an Wechsel- und Drehstromgeneratoren. Elektrotech Maschinenbau 32(25):615-618

2. Fleischmann L (1909) Ströme in Lagern und Wellen. Elektrische Kraftbetriebe und Bahnen 7(18):352-353

3. Kellner J (1911) Esetek az elektrotechnikai gyakorlatból. Csapágyáramok. (E: Cases from the electrical engineering practice. bearing currents). Elektrotech Mag 4(21):309-312

4. Ammann C, Reichert K, Joho R, Posedel Z (1988) Shaft voltages in generators with static excitation systems-problems and solution. Energy Convers IEEE Trans 3(2):409-419 
5. Kerszenbaum I (1992) Shaft currents in electric machines fed by solid-state drives. In: Industrial and commercial power systems technical conference Conference Record, Papers Presented at the 1992 Annual Meeting, S 71-79

6. Preisinger G (2002) Cause and effect of bearing currents in frequency converter driven electrical motors: Investigations of electrical properties of rolling bearings. Dissertation. Technische Universität Wien, Wien

7. Muetze A (2004) Bearing currents in inverter-fed AC-motors. Dissertation, Technische Universität Darmstadt. Shaker, Aachen

8. Zika T (2010) Electric discharge damaging in lubricated rolling contacts. Dissertation. Technische Universität Wien, Wien

9. Radnai B (2016) Wirkmechanismen bei spannungsbeaufschlagten Wälzlagern. Maschinenelemente und Getriebetechnik Berichte, Bd. 20. Technische Universität, Kaiserslautern. ISBN 978-3-95974029-6 (Dissertation)

10. Graf S, Sauer B (2020) Surface mutation of axial bearing raceways through the current passage in a mixed friction mode. IQPC-Component Innovation for E-drives, Berlin

11. Graf S, Sauer B (2021) Surface mutation of the bearing raceway during electrical current passage in mixed friction operation. Bear World J 5:137-147

12. Steinhilper W, Sauer B (2012) Konstruktionselemente des Maschinenbaus 2: Grundlagen von Maschinenelementen für Antriebsaufgaben, 7. Aufl. Springer Vieweg, Berlin. ISBN 978-3-642-24302-8.

13. Leidner M (2009) Kontaktphysikalische Simulation von Schichtsystemen. Dissertation. Darmstadt, Technische Universität Darmstadt

14. Boussinesq J (1885) Application des potentiels à l'etude de l'equilibre et du mouvement des solides élastiques. GauthierVillars, Paris

15. Bartel D (2010) Simulation von Tribosystemen. Vieweg+Teubner, Wiesbaden. ISBN 978-3-8348-1241-4.

16. Magyar B, Sauer B (2017) Methods for the simulation of the pressure, stress, and temperature distribution in the contact of fractal generated rough surfaces, in: Proceedings of the Institution of Mechanical Engineers, Part J. J Eng Tribol 231:489-502

17. Mueller M (2016) Zelluläre Automaten als Modellbildungswerkzeug für Fragestellungen aus der Technischen Mechanik. Schriftenreihe Institut für Dynamik und Schwingungen. TU Braunschweig, Braunschweig. ISBN 978-3-8440-4838-4.

18. Jacq C, N'elias D, Lormand G, Girodin D (2002) Development of a three-dimensional semi-analytical elastic-plastic contact code. J Tribol 124:653-667

19. Hammelmüller F, Zehetner C (2015) Increasing numerical efficiency in coupled Eulerian-Lagrangian metal forming simulations. Proceedings of the XIII International Conference on Computational Plasticity. Fundamentals and Applications, Barcelona

20. Abdelhafeez AM, Soo SL, Aspinwall D, Dowson A, Arnold D (2016) A coupled Eulerian lagrangian finite element model of drilling titanium and Aluminium alloys. SAE Int J Aerosp 9:198-207
21. Hongtao D, Yung SC (2012) A metallo-thermomechanically coupled analysis of orthogonal cutting of AISI 1045 steel. J Manuf Sci Eng 134:51014

22. Ansari MA, Samanta A, Behnagh RA, Ding H (2019) An efficient coupled Eulerian-Lagrangian finite element model for friction stir processing. Int J Adv Manuf Technol 101:1495-1508

23. Johansson H, Ollar J (2011) Polymer flow in package sealing process-a coupled Eulerian-lagrangian numerical approach. Master Thesis. University of Lund, Lund

24. Dassault Systemes (2017) Eulerian analysis, Abaqus manual SIMULIA user assistance

25. Dassault Systemes (2019) Modelling extreme deformation and fluid flow with Abaqus

26. Wagner M (2017) Lineare und nichtlineare FEM. Springer, Wiesbaden. ISBN 978-3-658-17865-9.

27. Bechev D, Weicker M (2020) Methodik zur praxisnahen Charakterisierung von elektrischen Schmierstoffeigenschaften zur Verbesserung der rechnerischen Vorhersage von Lagerströme. FVAForschungsvorhaben 650 II, Schädlicher Stromdurchgang II, Heft 1387. Forschungsvereinigung Antriebstechnik, Frankfurt am Main

28. Capan R, Gonda A, Bechev D, Sauer B (2019) The influence of lubricant conductivity on bearing currents in the case of rolling bearing greases. Mdpi Lubr. https://doi.org/10.3390/lubricants 7120108

29. Bechev D, Capan R, Gonda A, Sauer B (2019) Method for the investigation of the EDM breakdown voltage of grease and oil on rolling bearings. Bear World J 4:83-91

30. Tischmacher H (2017) Systemanalysen zur elektrischen Belastung von Wälzlagern bei umrichtergespeisten Elektromotoren. Dissertation. Institut für Antriebssysteme und Leistungselektronik, Universität Hannover, Hannover

31. Borlinghaus R (2016) Konfokale Mikroskopie in Weiß. Springer, Berlin. ISBN 978-3-662-49358-8.

32. Johnson GR, Cook WH (Hrsg) (1983) A constitutive model and data for metals subjected to large strains, high strain rates and high temperatures. Proceedings of the 7th International Symposium on Ballistics.

33. Lakshmana Rao C, Narayanamurthy V, Simha KRY (2016) Applied impact mechanics. Wiley, Chichester. ISBN 978-1-119-24180-5.

34. Bilici MA (2007) An investigation on the ballistic behaviour of alumina/aluminium armour structures. Dissertation. University of Ankara, Ankara

35. Gokhan O (2010) Numerical and experimental Invetigation of perforation of ST-37 steel plates by oblique impact. Master. University of Ankara, Ankara

36. Ellermann A (2012) Der Bauschingereffekt bei vergüteten, bainitischen und normalisierten Zuständen der Stähle $42 \mathrm{CrMoS} 4$ und 100Cr6. Dissertation. Universität Kassel, Kassel 